\title{
Multiple positive solutions to singular fractional differential equations with integral boundary conditions involving $p-q$-order derivatives
}

Han Wang ${ }^{1}$ and Jiqiang Jiang ${ }^{1 *}$ (D)

"Correspondence: qfijg@163.com

'School of Mathematical Sciences, Qufu Normal University, Qufu, China

\begin{abstract}
In this paper, we investigate the existence for a class of higher-order fractional differential equations with integral boundary value conditions involving $p-q$-order derivatives. As an application of the height functions on some special bounded sets, we obtain the existence of two positive solutions by means of the Leray-Schauder nonlinear alternative and cone expansion and cone compression fixed point theory. The nonlinearity may take negative infinity, and there may appear a singular phenomenon on both time and space variables.
\end{abstract}

MSC: 26A33; 34A08; 34B18

Keywords: Nonlocal fractional differential equations; Integral conditions; Semipositone; Singularity on space variable; Positive solutions

\section{Introduction}

The purpose of this paper is to obtain the existence of multiple positive solutions of the following singular fractional differential equations (FDEs for short) with $p-q$-order derivatives:

$$
\left\{\begin{array}{l}
D_{0^{+}}^{\alpha} x(t)+f(t, x(t))=0, \quad t \in(0,1), \\
x(0)=x^{\prime}(0)=x^{\prime \prime}(0)=\cdots=x^{(n-2)}(0)=0, \\
D_{0^{+}}^{p} x(1)=\lambda \int_{0}^{\eta} h(t) D_{0^{+}}^{q} x(t) d t,
\end{array}\right.
$$

where $D_{0^{+}}^{\alpha}$ is the standard Riemann-Liouville derivative of order $\alpha, n-1<\alpha \leq n, n \geq 3$, $h \in L^{1}[0,1]$ is nonnegative and may be singular at $t=0$ and $t=1, p, q \in \mathbb{R}, 1 \leq p \leq n-2$, $0 \leq q \leq p, \Delta=\Gamma(\alpha) / \Gamma(\alpha-p)\left(1-\lambda \int_{0}^{\eta} h(t) t^{\alpha-q-1} d t\right)>0, f(t, x)$ permits sign-changing and singularities at $t=0,1$ and/or $x=0$.

Nowadays FDEs nonlocal problems are of great interest because of their abilities to modeling complex phenomena in almost every field of science and technology. Many excellent works about FDEs nonlocal problems can be found in the literature (see [1, 3, 9-11, 13,

(c) The Author(s) 2019. This article is licensed under a Creative Commons Attribution 4.0 International License, which permits use, sharing, adaptation, distribution and reproduction in any medium or format, as long as you give appropriate credit to the original author(s) and the source, provide a link to the Creative Commons licence, and indicate if changes were made. The images or other third party material in this article are included in the article's Creative Commons licence, unless indicated otherwise in a credit line to the material. If material is not included in the article's Creative Commons licence and your intended use is not permitted by statutory regulation or exceeds the permitted use, you will need to obtain permission directly from the copyright holder. To view a copy of this licence, visit http://creativecommons.org/licenses/by/4.0/. 
$16,17,20,22,27,31]$ for instance). The existence of positive solutions for FDEs integral boundary problems (BVPs) with one or two parameters in boundary conditions is obtained by means of fixed point theory [5, 14, 22, 28]. The existence of positive solutions for integer-order differential equations with integral boundary conditions can be found in the literature (see $[8,13,15,18,24]$ for instance). As is well known, semipositone problems arise in bulking of mechanical systems, chemical reactions, astrophysics, combustion, management of natural resources, etc. Details are available in the works [2, 6, 15, 21, $23,24,26,29,32,34,35,37,39]$. Studying positive solutions for semipositone problems is more difficult than that for positive problems. Many methods used to deal with semipositone problems are, for example, variational methods, fixed point theory, subsuper solution methods, and degree theory.

Xu et al. [30] studied the existence and uniqueness of positive solutions for the fractional boundary value problem

$$
\left\{\begin{array}{l}
D_{0^{+}}^{\alpha} x(t)+h(t) f(t, x(t))=0, \quad t \in(0,1), n-1<\alpha \leq n, \\
x^{(k)}(0)=0, \quad 0 \leq k \leq n-2, \quad\left[D_{0^{+}}^{\beta} x\right]_{t=1}=0, \quad 1 \leq \beta \leq n-2,
\end{array}\right.
$$

where $n>3, D_{0^{+}}^{\alpha}$ is the standard Riemann-Liouville derivative, $f \in C([0,1] \times[0, \infty),[0, \infty))$ and $h \in C(0,1) \cap L(0,1)$ is nonnegative and may be singular at $t=0$ and/or $t=1$. In the case that $f$ is growing sublinearly, by means of fixed index theory and some spectral properties of associated linear integral operators the existence and uniqueness of positive solutions are obtained.

Salem [25] obtained the existence of pseudosolutions for the nonlinear multipoint boundary value problem of fractional order

$$
\left\{\begin{array}{l}
D_{0^{+}}^{\alpha} x(t)+q(t) f(t, x(t))=0, \quad \text { a.e. on }[0,1], \alpha \in(n-1, n], n \geq 2, \\
x(0)=x^{\prime}(0)=x^{\prime \prime}(0)=\cdots=x^{(n-1)}(0)=0, \quad x(1)=\sum_{i=1}^{m-2} \zeta_{i} x\left(\eta_{i}\right),
\end{array}\right.
$$

where $0<\eta_{1}<\eta_{2}<\cdots<\eta_{m-2}<1$, and $\zeta_{i}>0$ with $\sum_{i=1}^{m-2} \zeta_{i} \eta_{i}^{\alpha-1}<1$. It was assumed that $q$ is a real-valued continuous function and $f$ is a nonlinear Pettis-integrable function. Other relative papers on multipoint boundary value problem can be found in $[4,19,33,37]$.

In 2018, Zhang et al. [37] investigated the following singular differential equation with fractional derivative:

$$
\left\{\begin{array}{l}
D_{0^{+}}^{\alpha} x(t)+f(t, x(t))=0, \quad t \in(0,1), \\
x^{(k)}(0)=0, \quad 0 \leq k \leq n-2, \quad D_{0^{+}}^{p} x(1)=\sum_{i=1}^{m} a_{i} D_{0^{+}}^{q} x\left(\xi_{i}\right),
\end{array}\right.
$$

where $D_{0^{+}}^{\alpha}$ is the standard Riemann-Liouville derivative of order $\alpha, n-1<\alpha \leq n, n \geq 3$, $a_{i} \geq 0, i=1,2, \ldots, m\left(m \in \mathbb{N}^{+}\right), 0<\xi_{1}<\xi_{2}<\cdots<\xi_{m}<1, p, q \in \mathbb{R}, 1 \leq p \leq n-2$, and $0 \leq q \leq p$ with $\Delta=\Gamma(\alpha) / \Gamma(\alpha-p)\left(1-\sum_{i=1}^{m} a_{i} \xi_{i}^{\alpha-q-1}\right)>0$. The authors obtained the existence of triple positive solutions for fractional differential equations subject to multipoint boundary conditions by virtue of height functions on some special bounded sets. 
Zhang and Han [36] investigated the higher-order nonlocal fractional differential equations

$$
\left\{\begin{array}{l}
D_{0^{+}}^{\alpha} x(t)+f(t, x(t))=0, \quad t \in(0,1), n-1<\alpha \leq n \\
x^{(k)}(0)=0, \quad 0 \leq k \leq n-2, \quad x(1)=\int_{0}^{1} x(s) d A(s)
\end{array}\right.
$$

where $\alpha \geq 2, D_{0^{+}}^{\alpha}$ is the standard Riemann-Liouville derivative, $A$ is a function of bounded variation, and $\int_{0}^{1} x(s) d A(s)$ denotes the Riemann-Stieltjes integral. By applying the monotone iterative technique the existence and uniqueness of positive solutions were obtained, provided that $f(t, x)$ satisfies some growth conditions.

Inspired by the achievements mentioned, we consider the existence of multiple positive solutions of FDEs (1.1). In comparison with known results, this paper has some new features. Firstly, the nonlinearity $f$ may take negative infinity and change its sign. Secondly, the function $f(t, x)$ may be singular with respect to the time variable $t$ and/or the space variable $x$. Thirdly, the boundary conditions include an integral boundary condition involving $p-q$-order derivatives, quite different from those in $[25,30,36]$. Finally, the method in this paper is different from that in [38]. We devote ourselves to obtaining two existence results for BVP (1.1) by fixed point theory and the Leray-Schauder nonlinear alternative. Integration of height functions on special bounded sets is utilized to obtain the existence of positive solutions.

\section{Basic definitions and preliminaries}

This paper involves Banach spaces $X=C[0,1]$ and $L^{1}[0,1]$, the spaces of continuous functions and Lebesgue-integrable functions equipped with the norms $\|x\|=\max _{0 \leq t \leq 1}|x(t)|$ and $\|x\|_{1}=\int_{0}^{1}|x(t)| d t$.

Lemma 2.1 ([38], p. 13) Assume that $\Delta \neq 0$. Then for any $z \in C[0,1] \cap L^{1}[0,1]$, the solution of the boundary value problem

$$
\left\{\begin{array}{l}
D_{0^{+}}^{\alpha} x(t)+z(t)=0, \quad t \in(0,1), \\
x(0)=x^{\prime}(0)=x^{\prime \prime}(0)=\cdots=x^{(n-2)}(0)=0, \\
D_{0^{+}}^{p} x(1)=\lambda \int_{0}^{\eta} h(t) D_{0^{+}}^{q} x(t) d t,
\end{array}\right.
$$

satisfies

$$
x(t)=\int_{0}^{1} G(t, s) z(s) d s, \quad t \in(0,1),
$$

where

$$
G(t, s)=G_{1}(t, s)+G_{2}(t, s)
$$

with

$$
G_{1}(t, s)= \begin{cases}\frac{t^{\alpha-1}(1-s)^{\alpha-p-1}-(t-s)^{\alpha-p-1}}{\Gamma(\alpha)}, & 0 \leq s \leq t \leq 1 \\ \frac{t^{\alpha-1}(1-s)^{\alpha-p-1}}{\Gamma(\alpha)}, & 0 \leq t \leq s \leq 1\end{cases}
$$




$$
\begin{aligned}
& G_{2}(t, s)=\frac{\lambda t^{\alpha-1}}{\Delta} \int_{0}^{\eta} h(t) H(t, s) d t, \\
& H(t, s)= \begin{cases}\frac{t^{\alpha-q-1}(1-s)^{\alpha-p-1}-(t-s)^{\alpha-p-1}}{\Gamma(\alpha-q)}, & 0 \leq s \leq t \leq 1, \\
\frac{t^{\alpha-q-1}(1-s)^{\alpha-p-1}}{\Gamma(\alpha-q)}, & 0 \leq t \leq s \leq 1 .\end{cases}
\end{aligned}
$$

Definition 2.1 ([38], p. 13) A function $\zeta: K \longrightarrow[0,+\infty)$ is called a concave positive functional on a cone $K$ if

$$
\zeta(t x+(1-t) y) \geq t \zeta(x)+(1-t) \zeta(y), \quad x, y \in K, 0 \leq t \leq 1 .
$$

Lemma 2.2 ([37], p. 891) The Green function defined in Lemma 2.1 is a continuous function on $[0,1] \times[0,1]$ and satisfies the following conditions:

(i) $G(t, s) \leq J(s), t, s \in[0,1] \times[0,1]$, where

$$
\begin{aligned}
& J(s)=h_{1}(s)+\frac{\lambda}{\Delta} \int_{0}^{\eta} h(t) H(t, s) d t, \quad s \in[0,1], \\
& h_{1}(s)=(1-s)^{\alpha-p-1}\left(1-(1-s)^{p}\right) / \Gamma(\alpha), \quad s \in[0,1] ;
\end{aligned}
$$

(ii) $t^{\alpha-1} J(s) \leq G(t, s) \leq \sigma t^{\alpha-1}$, where

$$
\sigma=1 / \Gamma(\alpha)+\lambda \int_{0}^{\eta} h(t) t^{\alpha-q-1} d t /(\Delta \Gamma(\alpha-q)), \quad t, s \in[0,1] .
$$

Lemma 2.3 Let $w \in C[0,1]$ be a solution of

$$
\left\{\begin{array}{l}
D_{0^{+}}^{\alpha} x(t)+\phi(t)=0, \quad t \in(0,1), \\
x(0)=x^{\prime}(0)=x^{\prime \prime}(0)=\cdots=x^{(n-2)}(0)=0, \\
D_{0^{+}}^{p} x(1)=\lambda \int_{0}^{\eta} h(t) D_{0^{+}}^{q} x(t) d t,
\end{array}\right.
$$

where $\phi \in L^{1}[0,1], \phi(t)>0$. Then $w(t) \leq \sigma\|\phi\|_{1} t^{\alpha-1}, 0 \leq t \leq 1$.

Lemma 2.4 ([12], p. 94) Let $\Omega_{1}$ and $\Omega_{2}$ be two bounded open sets in a Banach space $X$ such $\theta \in \Omega_{1}$ and $\bar{\Omega}_{1} \subset \Omega_{2}$, and let $A: P \cap\left(\bar{\Omega}_{2} \backslash \Omega_{1}\right) \rightarrow P$ be a completely continuous operator, where $\theta$ denotes the zero element of $X$, and $P$ is a cone in $X$. Suppose that one of the two conditions holds:

(i) $\|A x\| \leq\|x\|, x \in P \cap \partial \Omega_{1} ;\|A x\| \geq\|x\|, x \in P \cap \partial \Omega_{2}$,

(ii) $\|A x\| \geq\|x\|, x \in P \cap \partial \Omega_{1} ;\|A x\| \leq\|x\|, x \in P \cap \partial \Omega_{2}$.

Then $A$ has a fixed point in $P \cap\left(\bar{\Omega}_{2} \backslash \Omega_{1}\right)$.

Lemma 2.5 ([7], P3) If $X$ is a Banach space, $D \subset X$ is convex with $\theta \subset D$, and $A: D \rightarrow D$ is a completely continuous operator, then either

(i) the set $B=\{x \in D: x=\lambda A(x), 0<\lambda<1\}$ is unbounded, or

(ii) $A$ has a fixed point.

\section{Main results}

Let $K=\left\{x \in X: x(t) \geq t^{\alpha-1}\|x\|\right.$ for $\left.t \in[0,1]\right\}$. Obviously, $K$ is a cone in $X$, and $(X, K)$ is a partially ordered Banach space.

The following hypotheses will be used throughout this paper: 
$\left(H_{1}\right) f \in C((0,1) \times(0,+\infty),(-\infty,+\infty))$, and there exists a function $\phi \in L^{1}[0,1], \phi(t)>0$, such that $f(t, x) \geq-\phi(t)$ for all $t \in(0,1)$ and $x>0$;

$\left(H_{2}\right) f^{*}(t, x)=f(t, x)+\phi(t)$ and $f^{*}(t, x) \leq q(t)[g(x)+h(x)]$ on $(0,1] \times(0,+\infty)$ with $g>0$ continuous and nonincreasing on $(0,+\infty), h>0$ continuous on $[0,+\infty), \frac{h}{g}$ nondecreasing on $(0,+\infty)$, and $q \in L^{1}[0,1]$ such that $q>0$ on $(0,1)$;

$\left(H_{3}\right)$ There exists $K_{0}>0$ such that $g(a b) \leq K_{0} g(a) g(b)$ for $a>0, b>0$;

$\left(H_{4}\right) a_{0}=\int_{0}^{1} q(s) g\left(s^{\alpha-1}\right) d s<+\infty$, and there exists $r>\sigma\|\phi\|_{1}$ such that

$$
\frac{r}{g\left(r-\sigma\|\phi\|_{1}\right)\left\{1+\frac{h(r)}{g(r)}\right\}}>\sigma a_{0} K_{0}
$$

$\left(H_{5}\right)$ For each $L>0$, there exists a positive function $\tau_{L} \in C[0,1]$ such that $f^{*}(t, x) \geq \tau_{L}(t)$ for $(t, x) \in[0,1] \times(0, L)$ and $\tau_{r}(t)>\phi(t)$, where $r$ is as in $\left(H_{4}\right)$;

$\left(H_{6}\right)$ There exist $R>r>\sigma\|\phi\|_{1}$ such that

$$
\int_{0}^{1} J(s) \varphi(s, r) d s<r
$$

and

$$
\int_{0}^{1} J(s) \psi(s, R) d s>R
$$

where

$$
\begin{aligned}
& \varphi(t, r)=\max \left\{f^{*}(t, x):\left(r-\sigma\|\phi\|_{1}\right) t^{\alpha-1} \leq x \leq r\right\}, \\
& \psi(t, R)=\min \left\{f^{*}(t, x):\left(R-\sigma\|\phi\|_{1}\right) t^{\alpha-1} \leq x \leq R\right\} .
\end{aligned}
$$

To establish the existence of a positive solution for BVP (1.1), we will concentrate on the following modified approximating BVP to overcome difficulties caused by singularities:

$$
\left\{\begin{array}{l}
D_{0^{+}}^{\alpha} x(t)+f^{*}(t, x(t)-w(t))=0, \quad t \in(0,1), \\
x(0)=x^{\prime}(0)=x^{\prime \prime}(0)=\cdots=x^{(n-2)}(0)=0, \\
D_{0^{+}}^{p} x(1)=\lambda \int_{0}^{\eta} h(t) D_{0^{+}}^{q} x(t) d t .
\end{array}\right.
$$

Define the operator $T$ by

$$
(T x)(t)=\int_{0}^{1} G(t, s) f^{*}(s, x(s)-w(s)) d s, \quad 0 \leq t \leq 1 .
$$

Lemma 3.1 Suppose that $\left(H_{1}\right)-\left(H_{4}\right)$ hold. Then for any $\sigma\|\phi\|_{1}<r<R$, the operator $T$ : $K \cap\left(\bar{\Omega}_{R} \backslash \Omega_{r}\right) \longrightarrow K$ is completely continuous.

Proof For any $0<t<1$ and $\sigma\|\phi\|_{1}<r<R$, since $x(t)-w(t) \geq\left(r-\sigma\|\phi\|_{1}\right) t^{\alpha-1}>0$, we have

$$
\left(r-\sigma\|\phi\|_{1}\right) t^{\alpha-1} \leq x(t)-w(t) \leq R .
$$


From this we get that

$$
\begin{aligned}
(T x) t & =\int_{0}^{1} G(t, s) f^{*}(s, x(s)-w(s)) d s \\
& \leq \sigma t^{\alpha-1} \int_{0}^{1} f^{*}(s, x(s)-w(s)) d s \\
& \leq \sigma \int_{0}^{1} q(s)[g(x(s)-w(s))+h(x(s)-w(s))] d s \\
& \leq \sigma \int_{0}^{1} q(s) g\left(\left(r-\sigma\|\phi\|_{1}\right) s^{\alpha-1}\right)\left\{1+\frac{h(R)}{g(R)}\right\} d s \\
& \leq \sigma a_{0} K_{0} g\left(r-\sigma\|\phi\|_{1}\right)\left\{1+\frac{h(R)}{g(R)}\right\}<+\infty .
\end{aligned}
$$

Therefore the operator $T: K \cap\left(\bar{\Omega}_{R} \backslash \Omega_{r}\right) \longrightarrow X$ is well defined. At the same time, we have the operator decomposition $T=N_{1} \circ N_{2}$, where

$$
\begin{aligned}
& \left(N_{1} x\right)(t)=\int_{0}^{1} G(t, s) x(s) d s, \quad t \in(0,1), x \in L_{1}[0,1], \\
& \left(N_{2} x\right)(t)=f^{*}(t, x(t)-w(t)), \quad \forall x \in K \cap\left(\bar{\Omega}_{R} \backslash \Omega_{r}\right) .
\end{aligned}
$$

By Lemma 2.2 we have

$$
\left(N_{1} x\right)(t)=\int_{0}^{1} G(t, s) x(s) d s \geq t^{\alpha-1} \int_{0}^{1} J(s) x(s) d s \geq t^{\alpha-1}\left\|N_{1} x\right\| .
$$

This shows that $N_{1}: L_{1}[0,1] \rightarrow K$. Furthermore, the operator $N_{1}: L_{1}[0,1] \longrightarrow K$ is completely continuous by the Arzelà-Ascoli theorem. To prove that the operator $T: K \cap$ $\left(\bar{\Omega}_{R} \backslash \Omega_{r}\right) \rightarrow K$ is completely continuous, we only need to prove that $N_{2}: K \cap\left(\bar{\Omega}_{R} \backslash \Omega_{r}\right) \rightarrow$ $L_{1}[0,1]$ is bounded and continuous. By assumptions $\left(H_{2}\right)-\left(H_{4}\right)$ we have

$$
\begin{aligned}
& \left|f^{*}(t, x(t)-w(t))\right| \leq K_{0} q(t) g\left(t^{\alpha-1}\right) g\left(r-\sigma\|\phi\|_{1}\right)\left\{1+\frac{h(R)}{g(R)}\right\}, \quad t \in[0,1], \\
& \left(r-\sigma\|\phi\|_{1}\right) t^{\alpha-1} \leq x(t)-w(t) \leq R .
\end{aligned}
$$

This shows that for $x \in K \cap\left(\bar{\Omega}_{R} \backslash \Omega_{r}\right)$,

$$
\begin{aligned}
\int_{0}^{1}\left(N_{2} x\right)(t) d t & =\int_{0}^{1} f^{*}(t, x(t)-w(t)) d t \\
& \leq a_{0} K_{0} g\left(r-\sigma\|\phi\|_{1}\right)\left\{1+\frac{h(R)}{g(R)}\right\}<+\infty
\end{aligned}
$$

Consequently, $N_{2}\left(K \cap\left(\bar{\Omega}_{R} \backslash \Omega_{r}\right)\right) \subset L_{1}[0,1]$ is bounded. Let

$$
x_{m}, x_{0} \in K \cap\left(\bar{\Omega}_{R} \backslash \Omega_{r}\right), \quad m=1,2, \ldots,
$$

and $\left\|x_{m}-x_{0}\right\| \rightarrow 0$. Then we have

$$
x_{m}(t)-x_{0}(t) \rightarrow 0, \quad t \in[0,1] .
$$


By $\left(H_{1}\right)$ we get

$$
f^{*}\left(t, x_{m}(t)-w(t)\right)-f^{*}\left(t, x_{0}(t)-w(t)\right) \longrightarrow 0, \quad \text { a.e. } t \in[0,1]
$$

By the Lebesgue dominated convergence theorem we have that $N_{2}: K \cap\left(\bar{\Omega}_{R} \backslash \Omega_{1}\right) \rightarrow$ $L_{1}[0,1]$ is continuous. Thus $T: K \cap\left(\bar{\Omega}_{R} \backslash \Omega_{r}\right) \longrightarrow K$ is completely continuous.

Theorem 3.1 Suppose that $\left(H_{1}\right)-\left(H_{6}\right)$ hold. Then BVP (1.1) has at least two positive solutions.

Proof For $x \in K \cap \partial \Omega_{r}$, we have $x(t) \geq r t^{\alpha-1}, t \in[0,1]$. Thus we get

$$
\left(r-\sigma\|\phi\|_{1}\right) t^{\alpha-1} \leq x(t)-w(t) \leq r, \quad 0<t<1 .
$$

By $\left(H_{6}\right)$ and Lemma 2.2 we have

$$
\begin{aligned}
\|T x\| & =\max _{0 \leq t \leq 1} \int_{0}^{1} G(t, s) f^{*}(s, x(s)-w(s)) d s \\
& \leq \int_{0}^{1} J(s) \varphi(s, r) d s<r .
\end{aligned}
$$

Obviously, $\|T x\| \leq\|x\|$ for $x \in K \cap \partial \Omega_{r}$. For $x \in K \cap \partial \Omega_{R}, x(t) \geq R t^{\alpha-1}, t \in[0,1]$. Thus we have

$$
R \geq x(t)-w(t) \geq\left(R-\sigma\|\phi\|_{1}\right) t^{\alpha-1} .
$$

By $\left(H_{6}\right)$ and Lemma 2.2 we also obtain

$$
\begin{aligned}
\|T x\| & =\max _{0 \leq t \leq 1} \int_{0}^{1} G(t, s) f^{*}(s, x(s)-w(s)) d s \\
& \geq \max _{0 \leq t \leq 1} \int_{0}^{1} J(s) f^{*}(s, x(s)-w(s)) d s \\
& \geq \int_{0}^{1} J(s) \psi(s, R) d s \\
& >R,
\end{aligned}
$$

that is, $\|T x\| \geq\|x\|$ for all $x \in K \cap \partial \Omega_{R}$. By Lemma 2.4 we have that $T$ has one fixed point $x_{1} \in K \cap\left(\bar{\Omega}_{R} \backslash \Omega_{r}\right)$. Therefore

$$
\begin{aligned}
& x_{1}(t) \geq\left\|x_{1}\right\| t^{\alpha-1} \geq r t^{\alpha-1}>\sigma\|\phi\|_{1} t^{\alpha-1} \geq w(t), \quad t \in[0,1] . \\
& x_{1}(t)=\int_{0}^{1} G(t, s) f^{*}\left(s, x_{1}(s)-w(s)\right) d s, \quad 0<t<1 .
\end{aligned}
$$

Consider the family of equations

$$
\left(T_{n} x\right)(t)=\int_{0}^{1} G(t, s) f_{n}^{*}(s, x(s)-w(s)) d s+\frac{1}{n}, \quad 0 \leq t \leq 1, n \in \mathbb{N}
$$


where

$$
f_{n}^{*}(t, x)= \begin{cases}f^{*}(t, x), & x \geq \frac{1}{n}, \\ f^{*}\left(t, \frac{1}{n}\right), & x<\frac{1}{n} .\end{cases}
$$

Similar to the proof of Lemma 3.1, we can show that the operator $T_{n}: K \cap \Omega_{r} \rightarrow K$ is completely continuous.

We consider

$$
x=\lambda T_{n} x+(1-\lambda) \frac{1}{n},
$$

that is,

$$
x(t)=\lambda \int_{0}^{1} G(t, s) f_{n}^{*}(s, x(s)-w(s)) d s+\frac{1}{n}, \quad t \in[0,1],
$$

where $\lambda \in[0,1]$. We claim that any fixed point of (3.12) for any $\lambda \in[0,1]$ must satisfy $\|x\| \neq r$. Otherwise, assume that $x$ is a fixed point of (3.12) for some $\lambda \in[0,1]$ such that $\|x\|=r$. Note that

$$
\begin{aligned}
x(t)-\frac{1}{n} & =\lambda \int_{0}^{1} G(t, s) f_{n}^{*}(s, x(s)-w(s)) d s \\
& \leq \lambda \int_{0}^{1} J(s) f_{n}^{*}(s, x(s)-w(s)) d s .
\end{aligned}
$$

Then we have

$$
\left\|x-\frac{1}{n}\right\| \leq \lambda \int_{0}^{1} J(s) f_{n}^{*}(s, x(s)-w(s)) d s .
$$

On the other hand, we get

$$
\begin{aligned}
x(t)-\frac{1}{n} & =\lambda \int_{0}^{1} G(t, s) f_{n}^{*}(s, x(s)-w(s)) d s \\
& \geq \lambda t^{\alpha-1} \int_{0}^{1} J(s) f_{n}^{*}(s, x(s)-w(s)) d s \\
& \geq t^{\alpha-1}\left\|x-\frac{1}{n}\right\| .
\end{aligned}
$$

By the choice of $n_{0}, \frac{1}{n} \leq \frac{1}{n_{0}}<r-\sigma\|\phi\|_{1}$. Hence we have

$$
x(t) \geq t^{\alpha-1}\left\|x-\frac{1}{n}\right\|+\frac{1}{n} \quad \geq t^{\alpha-1}\left(\|x\|-\frac{1}{n}\right)+\frac{1}{n} \geq r t^{\alpha-1}+\left(1-t^{\alpha-1}\right) \frac{1}{n} .
$$

Therefore

$$
\begin{aligned}
x(t)-w(t) & \geq r t^{\alpha-1}+\left(1-t^{\alpha-1}\right) \frac{1}{n}-\sigma\|\phi\|_{1} t^{\alpha-1} \\
& \geq\left(r-\sigma\|\phi\|_{1}-\frac{1}{n}\right) t^{\alpha-1}+\frac{1}{n}>\frac{1}{n}
\end{aligned}
$$


and

$$
\begin{aligned}
x(t)-w(t) & \geq r t^{\alpha-1}+\left(1-t^{\alpha-1}\right) \frac{1}{n}-\sigma\|\phi\|_{1} t^{\alpha-1} \\
& \geq\left(r-\sigma\|\phi\|_{1}\right) t^{\alpha-1}+\left(1-t^{\alpha-1}\right) \frac{1}{n} \\
& \geq\left(r-\sigma\|\phi\|_{1}\right) t^{\alpha-1} .
\end{aligned}
$$

For all $t \in[0,1]$, from condition $\left(H_{4}\right)$ we have that

$$
\begin{aligned}
x(t) & =\lambda \int_{0}^{1} G(t, s) f_{n}^{*}(s, x(s)-w(s)) d s+\frac{1}{n} \\
& =\lambda \int_{0}^{1} G(t, s) f^{*}(s, x(s)-w(s)) d s+\frac{1}{n} \\
& \leq \sigma t^{\alpha-1} \int_{0}^{1} f^{*}(s, x(s)-w(s)) d s+\frac{1}{n} \\
& \leq \sigma a_{0} K_{0} g\left(r-\sigma\|\phi\|_{1}\right)\left\{1+\frac{h(r)}{g(r)}\right\}+\frac{1}{n} .
\end{aligned}
$$

Therefore

$$
r=\|x\| \leq \sigma a_{0} K_{0} g\left(r-\sigma\|\phi\|_{1}\right)\left\{1+\frac{h(r)}{g(r)}\right\}+\frac{1}{n} .
$$

This is a contradiction to the choice of $n_{0}$, and the claim is proved. Now the LeraySchauder alternative principle guarantees that

$$
x(t)=\lambda \int_{0}^{1} G(t, s) f_{n}^{*}(s, x(s)-w(s)) d s+\frac{1}{n}
$$

has a fixed point $\bar{x}_{n}$ in $K \cap \Omega_{r}$.

Next, we claim that $\bar{x}_{n}(t)-w(t)$ has a uniform positive lower bound: there exists a constant $\delta>0$ such that

$$
\min _{t \in[0,1]}\left\{\bar{x}_{n}(t)-w(t)\right\} \geq \delta t^{\alpha-1}
$$

for all $n \in \mathbb{N}$. Since $\left(H_{6}\right)$ holds, there exists a continuous function $\tau_{r}(t)>0$ such that $f^{*}(t, x)>\tau_{r}(t)>\phi(t)$ for all $(t, x) \in[0,1] \times(0, r]$.

Since $\bar{x}_{n}(t)-w(t)<r$, we have

$$
\begin{aligned}
\bar{x}_{n}(t)-w(t) & =\int_{0}^{1} G(t, s) f_{n}^{*}\left(s, \bar{x}_{n}(s)-w(s)\right) d s+\frac{1}{n}-\int_{0}^{1} G(t, s) \phi(s) d s \\
& \geq \int_{0}^{1} G(t, s)\left(\tau_{r}(s)-\phi(s)\right) d s \\
& \geq t^{\alpha-1} \int_{0}^{1} J(s)\left(\tau_{r}(s)-\phi(s)\right) d s=\delta t^{\alpha-1},
\end{aligned}
$$

where $\delta=\int_{0}^{1} J(s)\left(\tau_{r}(s)-\phi(s)\right) d s$. 
By $\left(H_{1}\right)$ we have that $\bar{x}_{n}$ are bounded and equicontinuous on $[0,1]$. The Arzelà-Ascoli theorem implies that there exist a subsequence $N_{0}$ of $N$ and a function $x_{2}$ such that $\bar{x}_{n}$ converges to $x_{2}$ uniformly on $[0,1]$ as $n \rightarrow \infty$ through $N_{0}$. Since $\min _{t \in[0,1]}\left\{\bar{x}_{n}(t)-w(t)\right\} \geq \delta t^{\alpha-1}$ and $\left\|\bar{x}_{n}\right\|<r$, it follow that $x_{2}$ satisfies $\delta t^{\alpha-1} \leq x_{2}(t)-w(t)<r$ for all $t \in[0,1]$. Therefore $x_{2}$ is a positive solution of (3.4). Letting $n \rightarrow \infty$ on both sides, we have

$$
x_{i}(t)=\int_{0}^{1} G(t, s) f^{*}\left(s, x_{i}(s)-w(s)\right) d s, \quad i=1,2, t \in[0,1]
$$

Let $x_{i}^{\star}(t)=x_{i}(t)-w(t)$. Then from (3.13) it follows that $x_{i}^{\star}(t)(i=1,2)$ are positive solutions of BVP (1.1).

Remark 3.1 Suppose that $\left(H_{1}\right)-\left(H_{5}\right)$ and the following conditions are satisfied:

$$
\int_{0}^{1} J(s) \varphi\left(s, r_{i}\right) d s<r_{i}, \quad \int_{0}^{1} J(s) \varphi\left(s, R_{i}\right) d s>R_{i},
$$

where $R_{i}>r_{i}>\sigma\|\phi\|_{1}(i=1,2, \ldots, m)$. Then BVP (1.1) has at least $m+1$ positive solutions.

\section{An example}

Example 4.1 Consider the fractional differential equation

$$
\left\{\begin{array}{l}
D_{0^{+}}^{\frac{5}{2}} x(t)+f(t, x(t))=0, \quad t \in(0,1) \\
x(0)=x^{\prime}(0)=0, \quad x^{\prime}(1)=\frac{1}{3} \int_{0}^{\frac{1}{3}} t^{-\frac{1}{4}} D_{0^{+}}^{\frac{1}{2}} x(t) d t
\end{array}\right.
$$

where $f(t, x)=\frac{1}{10} t^{10}\left(\frac{1}{\sqrt{u}}+u^{\frac{7}{2}}\right)-\frac{1}{30} t^{10}$. It is clear that $\alpha=\frac{5}{2}, n=3, p=1, q=\frac{1}{2}, \lambda=\frac{1}{3}, \eta=\frac{1}{3}$, $h(t)=t^{-\frac{1}{4}}$.

By a simple computation $\Gamma(\alpha)=1.3294$ and $\lambda \int_{0}^{\eta} h(t) t^{\alpha-q-1} d t=0.0279<1$.

Clearly, $\left(H_{1}\right)$ holds for $\phi(t)=\frac{1}{30} t^{10}$.

After direct calculation, we have $\sigma=0.7714,\|\phi\|_{1}=0.0031$, and $\sigma\|\phi\|_{1}=0.0024$. Let $q(t)=\frac{1}{10} t^{10}, g(x)=\frac{1}{\sqrt{x}}, h(x)=x^{\frac{7}{2}}, K_{0}=0.1$. Then $\left(H_{2}\right)$ and $\left(H_{3}\right)$ are satisfied.

We take $r=1>0.0024=\sigma\|\phi\|_{1}$, and by direct calculation we have

$$
a_{0}=\int_{0}^{1} q(s) g\left(s^{\alpha-1}\right) d s=0.9978<+\infty
$$

and

$$
\frac{r}{g\left(r-\sigma\|\phi\|_{1}\right)\left\{1+\frac{h(r)}{g(r)}\right\}}=0.4994>0.0769=\sigma a_{0} K_{0} .
$$

Thus $\left(H_{4}\right)$ is verified. Then by Lemma 2.2 we get

$$
\begin{aligned}
\int_{0}^{1} J(s) \varphi(s, 1) d s \leq & \frac{1}{10} \int_{0}^{1}\left[\frac{(1-s)^{\alpha-p-1}\left(1-(1-s)^{p}\right)}{\Gamma(\alpha)}\right. \\
& \left.+\frac{\lambda(1-s)^{\alpha-p-1} \int_{0}^{\eta} h(t) t^{\alpha-q-1} d t}{\Delta \Gamma(\alpha-p)}\right]
\end{aligned}
$$




$$
\begin{aligned}
& \cdot s^{10} \max \left\{\left(\frac{1}{\sqrt{x}}+x^{\frac{7}{2}}\right): 0.9766 s^{\alpha-1} \leq x \leq 1\right\} d s \\
= & \frac{1}{10} \int_{0}^{1}\left[\frac{(1-s)^{\frac{1}{2}}\left(1-(1-s)^{1}\right)}{\Gamma\left(\frac{5}{2}\right)}+\frac{\frac{1}{3}(1-s)^{\frac{1}{2}} \int_{0}^{\frac{1}{3}} t^{\frac{3}{4}} d t}{\Delta \Gamma\left(\frac{3}{2}\right)}\right] \\
& \cdot s^{10} \max \left\{\left(\frac{1}{\sqrt{x}}+x^{\frac{7}{2}}\right): 0.9766 s^{\frac{3}{2}} \leq x \leq 1\right\} d s \\
\leq & \frac{1}{10} \int_{0}^{1}\left[0.7523(1-s)^{\frac{1}{2}}-0.7523(1-s)^{\frac{3}{2}}+0.215(1-s)^{\frac{1}{2}}\right] \\
& \cdot s^{10}\left(\frac{1}{\sqrt{0.9766}} s^{-\frac{3}{4}}+1\right) d s \\
\leq & \frac{1}{10} \times(0.5016-0.3009+0.1434)\left(\frac{1}{\sqrt{0.9766}}+1\right) \\
\leq & \frac{1}{10} \times 0.3441 \times 1.9883 \\
= & 0.0685<1
\end{aligned}
$$

which means that $\int_{0}^{1} J(s) \varphi(s, 1) d s<1$. Then by taking $\tau_{r}(t)=\frac{1}{15} t^{10}\left(H_{5}\right)$ is verified.

Since $\Gamma\left(\frac{5}{4}\right)=0.9064$ and $\Gamma\left(\frac{3}{4}\right)=1.2255$, taking $R=50$, we have

$$
\begin{aligned}
& \int_{0}^{1} J(s) \psi(s, R) d s \geq \frac{1}{10} \int_{0}^{1}\left[\frac{(1-s)^{\alpha-p-1}\left(1-(1-s)^{p}\right)}{\Gamma(\alpha)}\right. \\
& \left.+\frac{\lambda \int_{0}^{\eta} h(t)\left[t^{\alpha-q-1}(1-s)^{\alpha-p-1}-(t-s)^{\alpha-p-1}\right] d t}{\Delta \Gamma(\alpha-p)}\right] \\
& \cdot s^{10} \min \left\{\left(\frac{1}{\sqrt{x}}+x^{\frac{7}{2}}\right): 49.9976 s^{\alpha-1} \leq x \leq 50\right\} d s \\
& \geq \frac{1}{10} \int_{0}^{1} \frac{(1-s)^{\alpha-p-1}\left(1-(1-s)^{p}\right)}{\Gamma(\alpha)} \\
& \cdot s^{10} \min \left\{\left(\frac{1}{\sqrt{x}}+x^{\frac{7}{2}}\right): 49.9976 s^{\alpha-1} \leq x \leq 50\right\} d s \\
& =\frac{1}{10} \int_{0}^{1} \frac{(1-s)^{\frac{1}{2}}\left(1-(1-s)^{1}\right)}{\Gamma\left(\frac{5}{2}\right)} \\
& \cdot s^{10} \min \left\{\left(\frac{1}{\sqrt{x}}+x^{\frac{7}{2}}\right): 49.9976 s^{\frac{3}{2}} \leq x \leq 50\right\} d s \\
& \geq \frac{1}{10} \int_{0}^{1} \frac{(1-s)^{\frac{1}{2}}}{\Gamma\left(\frac{5}{2}\right)} \cdot s^{11}\left(\frac{1}{\sqrt{50}}+(49.9976)^{\frac{7}{2}} s^{\frac{21}{4}}\right) d s \\
& \geq \frac{(49.9976)^{\frac{7}{2}}}{10 \Gamma\left(\frac{5}{2}\right)} \int_{0}^{1}(1-s)^{\frac{1}{2}} s^{\frac{65}{4}} d s \\
& \geq \frac{(49.9976)^{\frac{7}{2}}}{10 \Gamma\left(\frac{5}{2}\right)} \times \frac{\Gamma\left(\frac{69}{4}\right) \Gamma\left(\frac{3}{2}\right)}{\Gamma\left(\frac{75}{4}\right)} \\
& \geq 0.0001 \times(49.9976)^{\frac{7}{2}}=88.8734>50 \text {. }
\end{aligned}
$$

Hence $\left(H_{6}\right)$ is checked. Therefore all conditions of Theorem 3.1 are satisfied, and by Theorem 3.1 the BVP (4.1) has at least two solutions. 


\section{Conclusion}

In this paper, we obtained several sufficient conditions for the existence of positive solutions for nonlinear fractional differential equation involving integral boundary conditions. Our results will be a useful contribution to the existing literature on fractional-order nonlocal differential equations.

\section{Acknowledgements}

The authors would like to thank the referee for his/her very important comments that improved the results and quality of the paper.

\section{Funding}

This work is supported financially by the National Natural Science Foundation of China (11871302), a Project of Shandong Province Higher Educational Science and Technology Program (J18KA217), Doctoral Scientific Research Foundation of Qufu Normal University and Youth Foundation of Qufu Normal University (BSQD20130140).

\section{Abbreviations}

BVP, Boundary value problems.

Availability of data and materials

Data sharing not applicable to this paper as no data sets were generated or analyzed during the current study.

\section{Competing interests}

The authors declare that they have no competing interests.

\section{Authors' contributions}

The study was carried out in collaboration of both authors. Both authors read and approved the final manuscript.

\section{Publisher's Note}

Springer Nature remains neutral with regard to jurisdictional claims in published maps and institutional affiliations.

Received: 6 September 2019 Accepted: 5 December 2019 Published online: 02 January 2020

\section{References}

1. Ahmad, B., Nieto, J.J., Alsaedi, A., Al-Hutami, H.: Existence of solutions for nonlinear fractional q-difference integral equations with two fractional orders and nonlocal four-point boundary conditions. J. Franklin Inst. 351, 2890-2909 (2014)

2. Bai, D., Henderson, J., Zeng, Y.: Positive solutions of discrete Neumann boundary value problems with sign-changing nonlinearities. Bound. Value Probl. 2015, 231 (2015)

3. Bai, Z:: On positive solutions of a nonlocal fractional boundary problem. Nonlinear Anal. 72, 916-924 (2010)

4. Bai, Z:: Solvability for a class of fractional $m$-point boundary value problem at resonance. Comput. Math. Appl. 62 , 1292-1302 (2011)

5. Cabada, A., Wang, G.: Positive solutions of nonlinear fractional differential equations with integral boundary value conditions. J. Math. Anal. 389, 403-411 (2012)

6. Cheng, W., Xu, J., Cui, Y.: Positive solutions for a system of nonlinear semipositone fractional q-difference equations with q-integral boundary conditions. J. Nonlinear Sci. Appl. 10, 4430-4440 (2017)

7. Fang, H., Song, M.: Existence results for fractional order impulsive functional differential equations with multiple delays. Adv. Differ. Equ. 2018, 139 (2018)

8. Feng, M., Li, P., Sun, S.: Symmetric positive solutions for fourth-order $n$-dimensional $m$-Laplace systems. Bound. Value Probl. 2018, 63 (2018)

9. Feng, M., Zhang, X., Ge, W.: New existence results for higher-order nonlinear fractional differential equation with integral boundary conditions. Bound. Value Probl. 2011, 720702 (2011)

10. Goodrich, C.S.: Existence and uniqueness of solutions to a fractional difference equations with nonlocal conditions. Comput. Math. Appl. 61, 191-202 (2011)

11. Goodrich, C.S.: On semipositone discrete fractional boundary value problems with non-local boundary conditions. J. Differ. Equ. Appl. 19, 1758-1780 (2013)

12. Guo, D., Lakshmikantham, V.: Nonlinear Problems in Abstract Cones. Notes and Reports in Mathematics in Science and Engineering. Academic Press, Boston (1988)

13. Infante, G., Pietramala, P., Tenuta, M.: Existence and localization of positive solutions for a nonlocal BVP arising in chemical reactor theory. Commun. Nonlinear Sci. Numer. Simul. 19, 2245-2251 (2014)

14. Jiang, J., Liu, L., Wu, Y.: Multiple positive solutions of singular fractional differential system involving Stieltjes integral conditions. Electron. J. Qual. Theory Differ. Equ. 2012, 43 (2012)

15. Jiang, J., Liu, L., Wu, Y.: Positive solutions for second-order differential equations with integral boundary conditions. Bull. Malays. Math. Sci. Soc. 37(3), 779-796 (2014)

16. Jiang, J., O'Regan, D., Xu, J., Cui, Y.: Positive solutions for a Hadamard fractional p-Laplacian three-point boundary value problem. Mathematics 7, 439 (2019). https://doi.org/10.3390/math7050439

17. Jiang, J., O'Regan, D., Xu, J., Fu, Z.: Positive solutions for a system of nonlinear Hadamard fractional differential equations involving coupled integral boundary conditions. J. Inequal. Appl. 2019, 204 (2019) 
18. Jiao, L., Zhang, X.: A class of second-order nonlocal indefinite impulsive differential systems. Bound. Value Probl. 2018, $163(2018)$

19. Khalil, H., Khan, R.A., Baleanu, D., Saker, S.H.: Approximate solution of linear and nonlinear fractional differential equations under m-point local and nonlocal boundary conditions. Adv. Differ. Equ. 2016, 177 (2016)

20. Kilbas, A., Srivastava, H., Trujillo, J.: Theory and Applications of Fractional Differential Equations. North-Holland Mathematics Studies, vol. 204. Elsevier, Amsterdam (2006)

21. Li, H., Sun, J.: Positive solutions of superlinear semipositone nonlinear boundary value problems. Comput. Math. Appl. 61, 2806-2815 (2011)

22. Li, P., Feng, M.: Denumerably many positive solutions for a $n$-dimensional higher-order singular fractional differential system. Adv. Differ. Equ. 2018, 145 (2018)

23. Pu, R., Zhang, X., Cui, Y., Li, P., Wang, W.: Positive solutions for singular semipositone fractional differential equation subject to multipoint boundary conditions. J. Funct. Spaces 2017, Article ID 5892616 (2017)

24. Qin, P., Feng, M., Li, P.: Positive solutions to one-dimensional quasilinear impulsive indefinite boundary value problems. Adv. Differ. Equ. 2018, 421 (2018)

25. Salem, H.A.H.: On the fractional order $m$-point boundary value problem in reflexive Banach spaces and weak topologies. J. Comput. Appl. Math. 224, 565-572 (2009)

26. Su, H., Liu, L., Wu, Y.: Positive solutions for a nonlinear second-order semipositone boundary value system. Nonlinear Anal. 71(7-8), 3240-3248 (2009)

27. Wang, Y., Jiang, J.: Existence and nonexistence of positive solutions for the fractional coupled system involving generalized p-Laplacian. Adv. Differ. Equ. 2017, 337 (2017)

28. Wang, Y., Liu, L., Wu, Y.: Positive solutions for a nonlocal fractional differential equation. Nonlinear Anal. 74(11), 3599-3605 (2011)

29. Wang, Y., Liu, L., Zhang, X., Wu, Y.: Positive solutions of a fractional semipositone differential system arising from the study of HIV infection models. Appl. Math. Comput. 258, 312-324 (2015)

30. Xu, J., Wei, Z., Dong, W.: Uniqueness of positive solutions for a class of fractional boundary value problems. Appl. Math. Lett. 25, 590-593 (2012)

31. Yan, R., Sun, S., Sun, Y., Han, Z.: Boundary value problems for fractional differential equations with nonlocal boundary conditions. Adv. Differ. Equ. 2013, 176 (2013)

32. Yao, Q.: Existence of $n$ solutions and/or positive solutions to a semipositone elastic beam equation. Nonlinear Anal. 66(1), 138-150 (2007)

33. Zhai, C., Hao, M.: Multi-point boundary value problems for a coupled system of nonlinear fractional differential equations. Adv. Differ. Equ. 2015, 147 (2015)

34. Zhang, G., Liu, S.: On a class of semipositone discrete boundary value problems. J. Math. Anal. Appl. 325, 175-182 (2007)

35. Zhang, L., Ma, R.: Bifurcation structure of an asymptotically linear second-order semipositone discrete boundary value problem. J. Shandong Univ. Nat. Sci. 49(3), 79-83 (2014)

36. Zhang, $X .$, Han, Y.: Existence and uniqueness of positive solutions for higher order nonlocal fractional differential equations. Appl. Math. Lett. 25, 555-560 (2012)

37. Zhang, X., Shao, Z., Zhong, Q., Zhao, Z.: Triple positive solutions for semipositone fractional differential equations $m$-point boundary value problems with singularities and $p-q$-order derivatives. Nonlinear Anal., Model. Control 23(4), 889-903 (2018)

38. Zhang, X., Zhong, Q.: Triple positive solutions for nonlocal fractional differential equations with singularities both on time and space variables. Appl. Math. Lett. 80, 12-19 (2018)

39. Zhao, Z:: Existence of positive solutions for $2 n$ th-order singular semipositone differential equations with Sturm-Liouville boundary conditions. Nonlinear Anal. 72(3-4), 1348-1357 (2010)

\section{Submit your manuscript to a SpringerOpen ${ }^{\circ}$ journal and benefit from:}

- Convenient online submission

- Rigorous peer review

- Open access: articles freely available online

- High visibility within the field

- Retaining the copyright to your article

Submit your next manuscript at $\boldsymbol{s p r i n g e r o p e n . c o m ~}$ 